\title{
Exact Solutions for a Class of Nonlinear PDE with Variable Coefficients Using ET and ETEM
}

\author{
Shifei Sun, Lina Chang, Hanze Liu \\ School of Mathematical Sciences, Liaocheng University, Liaocheng, China \\ Email: 345503418@qq.com, changlina2018@163.com,hnz_liu@aliyun.com
}

How to cite this paper: Sun, S.F., Chang, L.N. and Liu, H.Z. (2019) Exact Solutions for a Class of Nonlinear PDE with Variable Coefficients Using ET and ETEM. Journal of Applied Mathematics and Physics, 7, 26852700.

https://doi.org/10.4236/jamp.2019.711183

Received: October 16, 2019

Accepted: November 2, 2019

Published: November 5, 2019

Copyright $\odot 2019$ by author(s) and Scientific Research Publishing Inc. This work is licensed under the Creative Commons Attribution International License (CC BY 4.0).

http://creativecommons.org/licenses/by/4.0/

\begin{abstract}
In this article, by using the modified CK direct method, we give a relationship between the generalized fifth-order KDV equations with variable coefficients and the corresponding constant coefficients ones. Then, we construct the abundant travelling solutions by the extended trial equation method (ETEM) in terms of different functions, such as the elliptic functions, rational functions, hyperbolic functions and trigonometric functions. The extended trial equation method is powerful and can be used to other partial differential equations and more research can be done by this method.
\end{abstract}

\section{Keywords}

Equivalence Transformation, Extend trial Equation Method,

Higher Order Partial Differential Equation, Travelling Wave Solution

\section{Introduction}

It is well known that the exact solutions of partial differential equations are an important problem in nonlinear science; the travelling wave solutions of partial differential equations with variable coefficients are always playing an important role in studying the long-time behavior of solutions and understanding the complex nonlinear fluctuations. Especially in the multifarious real physical background such as the field of nonlinear optical crystal and plasma, nonlinear partial differential equations (PDEs) with variable coefficients can often provide realistic and powerful models than the corresponding constant coefficients ones when the inhomogeneities of media are considered. There are lots of studies have been conducted with different types of the PDEs, such as the modified trigonometric functions series [1] [2], the $G^{\prime} / G$ expand method [3] [4], the first integral method [5] [6], the modified CK direct method [7] [8] [9], the $\exp (-\phi(\xi))$ method [10] [11], modified simple equation method [12] [13], infinite series 
method [14] [15], the Lie symmetry analysis method [16]-[21] and the extended trial equation method [22] [23]. In this paper, we will use the modified CK direct method and the extended trial equation method (ETEM) to discuss the exact solutions for the following generalized fifth-order nonlinear partial differential KDV equation [24] [25] [26] [27] with time-dependent variable coefficients of the dispersion:

$$
\frac{\partial}{\partial t} u=-\alpha(t) u^{2} \frac{\partial}{\partial x} u-\beta(t) \frac{\partial^{2} u}{\partial x^{2}} \frac{\partial u}{\partial x}-F(t) u \frac{\partial^{3} u}{\partial x^{3}}-G(t) \frac{\partial^{5} u}{\partial x^{5}} .
$$

here the derivative $u_{t}$ represents the time evolution of a travelling wave as it travels in a certain direction, the function of nonlinear terms $u^{2} u_{x}, u_{x} u_{x x}$ and $u u_{x x x}$ are to collect the waves and the function of the linear term $u_{x x x x x}$ is to disperse the waves, respectively, $\alpha(t), \beta(t), F(t), G(t)$ are arbitrary smooth functions of $t$. When $\alpha(t), \beta(t), F(t), G(t)$ be constant, the Equation (1) be the generalized fifth-order KDV equation. What's more, we can get many other famous fifth-order partial differential equations by taking different values of the parameters:

Sawada-Kotera (SK) equation: when $\beta=F=5, \alpha=\frac{F^{2}}{2}, G=1$

$$
u_{t}+5 u^{2} u_{x}+5 u_{x} u_{x x}+5 u u_{x x x}+u_{x x x x x}=0 .
$$

Caudrey-Dodd-Gibbon (CDG) equation: when $\beta=F=30, \alpha=\frac{F^{2}}{5}, G=1$

$$
u_{t}+180 u^{2} u_{x}+30 u_{x} u_{x x}+30 u u_{x x x}+u_{x x x x x}=0 .
$$

Lax equation: when $\beta=2 F, \alpha=\frac{3}{10} F^{2}, G=1$

$$
u_{t}+30 u^{2} u_{x}+20 u_{x} u_{x x}+10 u u_{x x x}+u_{x x x x x}=0 .
$$

Kaup-Kuperschmidt (KK) equation: when $F=10, \beta=\frac{5}{2} F, \alpha=\frac{F^{2}}{2}, G=1$

$$
u_{t}+50 u^{2} u_{x}+25 u_{x} u_{x x}+10 u u_{x x x}+u_{x x x x x}=0 .
$$

Ito equation: when $F=3, \beta=2 F, \alpha=\frac{2 F^{2}}{9}, G=1$

$$
u_{t}+2 u^{2} u_{x}+6 u_{x} u_{x x}+3 u u_{x x x}+u_{x x x x x}=0 .
$$

These equations can describe motions of waves in shallow water under gravity and in a one-dimensional nonlinear lattice. It is an important mathematical model with wide applications in quantum mechanics and nonlinear optics. Until now, a great number of authors have used lots of methods to solve these PDEs, Alvaro and $\mathrm{H}$. Salas use the exp function method to obtain some exact solutions of Equation (1), Abdul-Majid Wazwaz obtained some new solition solutions of the modified form of fifth-order KDV equation (fKdV). In 2014, F. I. Leite considered the new concepts of self-adjoint equations formulated by Ibragimov and Gandarias are applied to the fifth-order evolution KDV equations. In 1989, Clarkson and Kruskal put forward the CK direct method for the first time, and then 
Lou modified the CK direct method and proposed a simple method which called the modified CK direct method. Furthermore, many scholars have studied the nonlinear equations with this method. In this paper, a new ETEM method is applied to the variable coefficient KDV equation which is reduced by CK method for the first time, and a series of new exact solutions are obtained.

The aim of this research is to establish an equivalence relation between the variable coefficients PDE and the corresponding constant coefficients one, and use a variety of distinct trail equations to construct some new travelling wave solutions of the constant coefficients equation. In this way, we can obtain a series of new exact solutions of the variable-coefficient equation by the relation between the variable-coefficient equation and the constant-coefficient equation, such as the trigonometric function solution, soliton solution, periodic solution and rational solution. In section 2 , the equivalence relation between the fifth-order KDV equation with variable coefficient and the corresponding constant coefficient equation is given by using the modified CK direct method. In section 3 , the ETEM is briefly introduced. In section 4, we obtain some new types of travelling wave solutions to Equation (1). In section 5, the more possibility of ETEM method for solving partial differential equations is discussed. In section 6 , the conclusion and discussion are given.

\section{Equivalence Transformations (ETs) of Generalized Fifth-Order KDV Equation}

In this section, we will use the modified CK direct method to look for the equivalence transformations between Equation (1) and the corresponding following equation.

$$
u_{t}+a u^{2} u_{x}+b u_{x} u_{x x}+c u u_{x x x}+d u_{x x x x x}=0,
$$

where $a, b, c, d$ are arbitrary constants. Suppose that Equation (1) has the following solution:

$$
u(x, t)=A+B U(X, T),
$$

where $A=A(x, t), B=B(x, t), X=X(x, t), T=T(x, t)$ are functions can be determined by requiring $U=U(X, T)$ satisfy the transformation $\{x, t, u\} \rightarrow\{X, T, U\}$. in other words, we let $\{X, T, U\}$ to satisfied the corresponding constant coefficients equation of Equation (1) as following

$$
U_{T}+a U^{2} U_{X}+b U_{X} U_{X X}+c U U_{X X X}+d U_{X X X X X}=0 .
$$

Then substituting Equation (3) into Equation (1), requiring $U=U(X, T)$ and $U_{T}$ satisfied Equation (4), we can collect the coefficients of $U$, letting their derivatives to be zero, we have

$$
\begin{gathered}
G B X_{x x x x x}-d B T_{t}=0, \\
F B^{2} X_{x}^{3}-c B T_{t}=0, \\
\cdots \\
F B X_{x}^{3} A+15 G B X_{x} X_{x x}^{2}+10 G B X_{x}^{2} X_{x x x}=0,
\end{gathered}
$$




$$
\begin{gathered}
10 G B X_{x x} X_{x x x}+5 G B X_{x} X_{x x x x}+3 F B X_{x} X_{x x} a=0, \\
\beta B X_{x x} A_{x}+F B X_{x x x} A+G B X_{x x x x x}+B X_{t}=0, \\
G A_{x x x x x}+A_{x} \alpha A^{2}+\beta A_{x x} A_{x}+F A_{x x x} A+A_{t}=0 .
\end{gathered}
$$

Solving above equations, we have

$$
\begin{gathered}
A=0, B=C_{0}, F=\frac{c T_{t}}{C_{2}^{3} C_{0}}, G=d \frac{T_{t}}{C_{2}^{5}}, X=C_{2} X+C_{3}, \\
\alpha=a \frac{T_{t}}{C_{2} C_{0}^{2}}, \beta=b \frac{T_{t}}{C_{2}^{3} C_{0}},
\end{gathered}
$$

where $T_{t}$ is an arbitrary function of $t, C_{1}, C_{2}, C_{3}$ are arbitrary constants. Then with Equation (6), we can obtain new travelling wave exact solutions for the Equation (1) as follows:

$$
u=C_{0} U\left(C_{2} x+C_{3}, T(t)\right)
$$

If $U(X, T)$ is the solution of fifth-order KDV equation with constant coefficients, then $u(x, t)$ also is a solution of Equation (1).

\section{Description of the Extend Trial Equation Method}

In this section, we introduce the extend trial equation method (ETEM) as follows.

Step1. Firstly, we consider the following nonlinear partial differential equation:

$$
F_{1}\left(u, u_{t}, u_{x}, u_{x x}, u_{x t}, u_{t t}, u_{x x x}, \cdots\right)=0,
$$

In order to look for the solutions of Equation (1), we make the travelling wave transformation

$$
u(x, t)=U(\xi),
$$

where $\xi=x-c t$, and $c$ is an arbitrary constant.

Then the Equation (8) can be converted to an ordinary differential equation as following

$$
F_{2}\left(U, U_{\xi}, U_{\xi \xi}, U_{\xi \xi \xi}, \cdots\right)=0 .
$$

Step 2. We suppose that the exact solution of Equation (9) is of the form

$$
u=\sum_{i=1}^{\delta} \tau_{i} Y^{i}
$$

where $\tau_{i}(i=1,2,3, \cdots, \delta)$ are arbitrary constant, and $Y^{i}$ satisfied the following condition

$$
\left(Y^{\prime}\right)^{2}=\Lambda(Y)=\frac{\phi(Y)}{\psi(Y)}=\frac{\xi_{0}+\xi_{1} Y+\xi_{2} Y^{2}+\cdots+\xi_{\theta} Y^{\theta}}{\zeta_{0}+\zeta_{1} Y+\zeta_{2} Y^{2}+\cdots+\zeta_{\varepsilon} Y^{\varepsilon}},
$$

where $\xi_{\theta}$ and $\zeta_{\varepsilon}$ are arbitrary positive integers to be determined later.

Step 3. According to the balance principle we can determine a relation of $\theta$, $\varepsilon$ and $\delta$. We can take some different values of $\theta, \varepsilon$ and $\delta$ and the solution 
(10) can be written as a series of infinity solutions.

Step 4. Substituting Equation (10) and Equation (11) into Equation (9), letting the coefficients of $Y$ to be zero, by solving the system of algebraic equations by some software like Maple and Mathematica, we can figure out the values of $\xi_{\theta}, \zeta_{\varepsilon}$ and $\tau_{\delta}$, then reducing Equation (11) to the elementary form as follows:

$$
\pm\left(\xi-\eta_{0}\right)=\int \frac{1}{\sqrt{\Lambda(Y)}} \mathrm{d} Y=\int \sqrt{\frac{\psi(Y)}{\phi(Y)}} \mathrm{d} Y,
$$

where $\eta_{0}$ is an arbitrary constant.

Using a complete discrimination system for polynomial to classify the different forms of Equation (12), we can write the travelling wave solutions respectively. In this paper, the solutions of PDEs with variable coefficients also can be written respectively by the conclusion we got in the last section. Moreover, this method is appropriate for lots of other PDEs which can be discussed as follows.

\section{ETEM to Generalized Fifth-Order KDV Equation}

In this section, we discuss the generalized fifth-order KDV equation by using extend trial equation method.

$$
u_{t}+a u^{2} u_{x}+b u_{x} u_{x x}+c u u_{x x x}+d u_{x x x x x}=0 .
$$

Using the travelling wave transformation

$u(x, t)=U(\xi)$, where $\xi=x-C t$,

Equation (13) turns into the following ordinary differential equation, then integrate the equation once:

$$
C_{1}-C U+\frac{a}{3} U^{3}+\frac{b-c}{2}\left(U^{\prime}\right)^{2}+c U U^{\prime \prime}+d U^{(4)}=0
$$

where $C_{1}$ is the integration constant. Substituting Equations (10) and (11) into Equation (14)

$$
\begin{gathered}
U=\tau_{\delta} Y^{\delta}+\tau_{\delta-1} Y^{\delta-1}+\cdots+\tau_{0}, \\
U^{3}=\tau_{\delta}^{3} Y^{3 \delta}+\tau_{\delta-1}^{3} Y^{3 \delta-3}+\cdots+\tau_{0}^{3}, \\
U^{\prime}=\delta \tau_{\delta} Y^{\delta-1} Y^{\prime}+\cdots=X_{1}(\tau, \theta, \delta, \varepsilon) Y^{\frac{2 \delta+\theta-\varepsilon-2}{2}}+\cdots, \\
U^{(4)}=X_{2}(\tau, \theta, \delta, \varepsilon) Y^{\delta+2 \theta-2 \varepsilon-4}+\cdots .
\end{gathered}
$$

Using the balance principle, we find the highest degree terms of two Equations (15) and (16) should be the same, so we have

$$
\delta=\theta-\varepsilon-2 .
$$

By assuming and assigning the variables that satisfy the above conditions, we can obtain the traveling wave solution of the Equation (13) in many cases.

If $\varepsilon=0, \theta=3$ and $\delta=1$, then

$$
\begin{gathered}
U=\tau_{0}+\tau_{1} Y, \\
U^{\prime}=\tau_{1} \sqrt{\frac{\xi_{0}+\xi_{1} Y+\xi_{2} Y^{2}+\xi_{3} Y^{3}}{\zeta_{0}}},
\end{gathered}
$$




$$
\begin{gathered}
U^{\prime \prime}=\tau_{1} \frac{\left(\xi_{1}+2 \xi_{2} Y+3 \xi_{3} Y^{2}\right)}{2 \zeta_{0}}, \\
U^{\prime \prime \prime}=\tau_{1} \frac{\sqrt{\xi_{0}+\xi_{1} Y+\xi_{2} Y^{2}+\xi_{3} Y^{3}}\left(\xi_{2}+3 \xi_{3} Y\right)}{\zeta_{0}^{\frac{3}{2}}}, \\
U^{(4)}=\tau_{1} \frac{15 \xi_{3}^{2} Y^{3}+15 \xi_{2} \xi_{3} Y^{2}+9 \xi_{1} \xi_{3} Y+2 \xi_{2}^{2} Y+6 \xi_{0} \xi_{3}+\xi_{1} \xi_{2}}{2 \zeta_{0}^{2}},
\end{gathered}
$$

where $\zeta_{0} \xi_{3} \neq 0$.

Substituting Equation (17) into Equation (14), collecting the coefficients of $Y$ and solving these algebraic equation systems respectively, we can obtain:

$$
\begin{gathered}
\xi_{3}=L_{1} \zeta_{0} \tau_{1}, \\
\xi_{2}=-\frac{\zeta_{0} \tau_{0}\left(3 L_{1} c+2 a\right)}{L_{2}}, \\
\xi_{1}=\frac{\zeta_{0}\left(-9 L_{1}^{2} d c^{2} \tau_{0}^{2}+3 L_{1} L_{2} c^{2} \tau_{0}^{2}-12 L_{1} a d c \tau_{0}^{2}-L_{2}^{2} a \tau_{0}^{2}+2 L_{2} a c \tau_{0}^{2}-4 d a^{2} \tau_{0}^{2}+C L_{2}^{2}\right)}{\tau_{1} L_{2}^{2}\left(9 d L_{1}+b\right)} \\
\xi_{0}(\text { See Appendixes A1) }
\end{gathered}
$$

where $L_{1}= \pm \frac{-2 c-b+\sqrt{-40 a d+b^{2}+4 b c+4 c^{2}}}{30 d}$,

$L_{3}=54 L_{1}^{2} d^{2}+15 L_{1} d b-9 L_{1} d c+b^{2}-b c, L_{2}=15 d L_{1}+c+b$. We can use Equation (18) and Equation (12) to get

$$
\pm\left(\xi-\eta_{0}\right)=\int \frac{1}{\sqrt{\Lambda(Y)}} \mathrm{d} Y=\int \sqrt{\frac{\xi_{3} Y^{3}+\xi_{2} Y^{2}+\xi_{1} Y+\xi_{0} Y}{\zeta_{0}}} \mathrm{~d} Y .
$$

Then, we will discuss some of the special form of solutions of Equation (14) as follow.

If Equation (19) can be written as following form:

$$
\begin{aligned}
& \xi_{3} Y^{3}+\xi_{2} Y^{2}+\xi_{1} Y+\xi_{0} Y \\
& =L_{1} \tau_{1} Y^{3}-\frac{\tau_{0}\left(3 L_{1} c+2 a\right)}{L_{2}} Y^{2} \\
& +\frac{\left(-9 L_{1}^{2} d c^{2} \tau_{0}^{2}+3 L_{1} L_{2} c^{2} \tau_{0}^{2}-12 L_{1} a d c \tau_{0}^{2}-L_{2}^{2} a \tau_{0}^{2}+2 L_{2} a c \tau_{0}^{2}-4 d a^{2} \tau_{0}^{2}+C L_{2}^{2}\right)}{\tau_{1} L_{2}^{2}\left(9 d L_{1}+b\right)} Y \\
& +2 \zeta_{0}\left(-6 d \tau_{0}^{3} L_{2}^{2} a^{2}-L_{2}^{3} a b \tau_{0}^{3}+3 C L_{2}^{3} b \tau_{0}-27 C_{1} L_{1} L_{2}^{3} d-9 c^{3} \tau_{0}^{3} L_{2}^{2} L_{1}+3 a c \tau_{0}^{3} L_{2}^{3}\right. \\
& -6 a c^{2} \tau_{0}^{3} L_{2}^{2}-3 c \tau_{0} L_{2}^{3} C-81 d^{2} \tau_{0}^{3} L_{1}^{3} c^{3}-24 d^{2} \tau_{0}^{3} a^{3}-3 C_{1} L_{2}^{3} b+6 d \tau_{0} C L_{2}^{2} a \\
& -9 L_{1} L_{2}^{3} d a \tau_{0}^{3}+27 C L_{1} L_{2}^{3} d \tau_{0}+54 c^{3} \tau_{0}^{3} L_{2} L_{1}^{2} d+24 c \tau_{0}^{3} L_{2} d a^{2}-162 d^{2} \tau_{0}^{3} L_{1}^{2} c^{2} a \\
& \left.-108 d^{2} \tau_{0}^{3} L_{1} c a^{2}+72 c^{2} \tau_{0}^{3} L_{1} L_{2} a d-9 d \tau_{0}^{3} L_{1} L_{2}^{2} a c+9 d \tau_{0} C L_{1} L_{2}^{2} c\right) /\left(3 L_{2}^{3} \tau_{1}^{2} L_{3}\right) \\
& =\left(Y-\alpha_{1}\right)^{3} .
\end{aligned}
$$

where $\alpha_{1}$ is an arbitrary constant. Collecting the coefficients of $Y$ of Equation (20), let all coefficients to be zero, we can get the following results 


$$
\begin{gathered}
\tau_{1}=\frac{1}{L_{1}}, \tau_{0}=\frac{3 \alpha_{1} L_{2}}{3 c L_{1}+3 a}, \\
C=3 \alpha_{1}^{2}\left(135 L_{1}^{3} d c^{2}-18 L_{1}^{2} L_{2} c^{2}+180 L_{1}^{2} a d c+9 L_{1}^{2} b c^{2}+6 a L_{1} L_{2}^{2}-12 a c L_{1} L_{2}\right. \\
\left.+60 L_{1} d a^{2}+12 L_{1} a b c+4 a^{2} b\right) /\left(2 L_{1}\left(3 L_{1} c+2 a\right)\right), \\
C_{1} \quad \text { (See Appendixes A2) }
\end{gathered}
$$

In this family, Equations (18), (19) and (21) lead to get

$$
\xi_{3}=\zeta_{0}, \xi_{2}=-3 \alpha_{1} \zeta_{0}, \xi_{1}=3 \alpha_{1}^{2} \zeta_{0}, \xi_{0}=-\alpha_{1}^{3} \zeta_{0} .
$$

And

$$
\pm\left(\xi-\eta_{0}\right)=\int \frac{1}{\sqrt{\Lambda(Y)}} \mathrm{d} Y=\int \frac{\mathrm{d} Y}{\left(Y-\alpha_{1}\right)^{\frac{3}{2}}}=-\frac{2}{\sqrt{Y-\alpha_{1}}} .
$$

By Equation (23), (21), we can get the rational exact solution of Equation (14) as following

$$
\begin{gathered}
U(\xi)=\frac{3 \alpha_{1} L_{2}}{3 c L_{1}+3 a}+\frac{1}{L_{1}}\left[\alpha_{1}+\frac{4}{\left(\xi-\eta_{0}\right)^{2}}\right] . \\
u(x, t)=\frac{3 \alpha_{1} L_{2}}{3 c L_{1}+3 a}+\frac{1}{L_{1}}\left[\alpha_{1}+\frac{4}{\left(x-C t-\eta_{0}\right)^{2}}\right],
\end{gathered}
$$

where

$$
\begin{aligned}
C= & 3 \alpha_{1}^{2}\left(135 L_{1}^{3} d c^{2}-18 L_{1}^{2} L_{2} c^{2}+180 L_{1}^{2} a d c+9 L_{1}^{2} b c^{2}+6 a L_{1} L_{2}^{2}-12 a c L_{1} L_{2}\right. \\
& \left.+60 L_{1} d a^{2}+12 L_{1} a b c+4 a^{2} b\right) /\left(2 L_{1}\left(3 L_{1} c+2 a\right)\right),
\end{aligned}
$$

Hence, with the relation of Equation (7) obtained above, Equation (1) have the following form of rational travelling wave solution

$$
\begin{aligned}
u(x, t) & =C_{0} \frac{3 \alpha_{1}\left(15 d\left( \pm \frac{-2 c-b+\sqrt{-40 a d+b^{2}+4 b c+4 c^{2}}}{30 d}\right)+c+b\right)}{3 c\left( \pm \frac{-2 c-b+\sqrt{-40 a d+b^{2}+4 b c+4 c^{2}}}{30 d}\right)+3 a} \\
& +C_{0} \frac{1}{\left( \pm \frac{-2 c-b+\sqrt{-40 a d+b^{2}+4 b c+4 c^{2}}}{30 d}\right)}\left(\alpha_{1}+\frac{4}{\left(X-C T-\eta_{0}\right)^{2}}\right),
\end{aligned}
$$

where $X=C_{2} x+C_{3}, T$ is an arbitrary function of $t$ (Figure 1 and Figure 2).

1) In the following, we chose some new coefficients to make it easier to calculate

$$
\begin{gathered}
\xi_{3}=L_{1} \zeta_{0} \tau_{1}, \xi_{2}=L_{4} \zeta_{0} \tau_{0}, \\
\xi_{1}=\left(2\left(-L_{4}^{2} d \tau_{0}^{2}+L_{4} c \tau_{0}^{2}-a \tau_{0}^{2}+C\right) \zeta_{0}\right) /\left(\tau_{1}\left(9 d L_{4}+b\right)\right), \\
\xi_{0}=2 \zeta_{0}\left(-9 a d L_{1} \tau_{0}^{3}-a b \tau_{0}^{3}+27 d C L_{1} \tau_{0}+3 b C \tau_{0}-27 d L_{1} C_{1}-3 b C_{1}+6 c d L_{4}^{2} \tau_{0}^{3}\right. \\
\left.-3 c^{2} L_{4} \tau_{0}^{3}+3 a c \tau_{0}^{3}-3 c C \tau_{0}-3 d^{2} L_{4}^{3} \tau_{0}^{3}-3 a d L_{4} \tau_{0}^{3}+3 d C L_{4} \tau_{0}\right) /\left(3 \tau_{1}^{2} L_{3}\right) .
\end{gathered}
$$




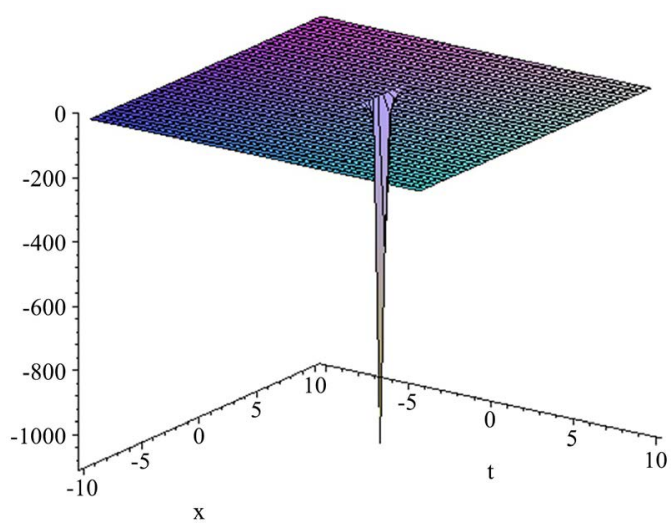

Figure 1. The three-dimensional picture of the exact solution (25) of Equation (1), and its projection at $b=3, c=2, a=d=1, \eta_{0}=3$ and $\alpha=11$.

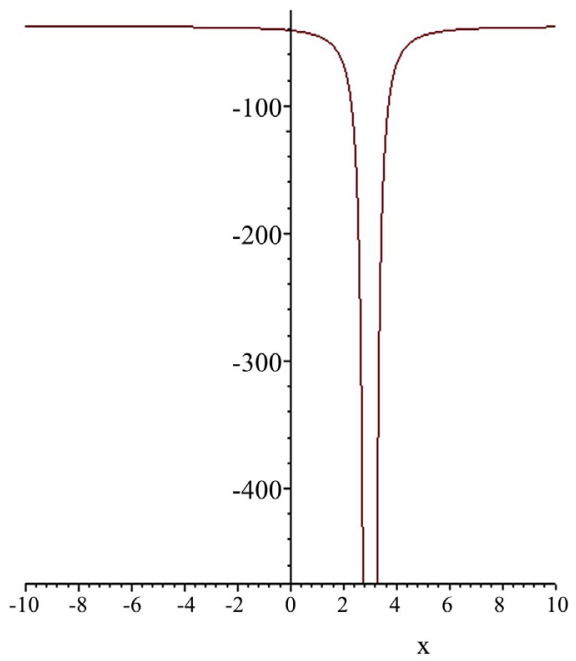

Figure 2. The two-dimensional picture of the equation exact solution (25) of Equation (1), and its projection at $t=0$. When $b=3, c=2, a=d=1, \eta_{0}=3$ and $\alpha=11$.

where

$$
\begin{gathered}
L_{1}= \pm \frac{-2 c-b+\sqrt{-40 a d+b^{2}+4 b c+4 c^{2}}}{30 d} \\
L_{3}=54 L_{1}^{2} d^{2}+15 L_{1} d b-9 L_{1} d c+b^{2}-b c \\
L_{4}=\left(3 c L_{1}+2 a\right) /\left(15 d L_{1}+b+c\right) .
\end{gathered}
$$

If Equation (19) can be written as following form:

$$
\begin{aligned}
& L_{1} \tau_{1} Y^{3}+L_{4} \tau_{0} Y^{2}+\frac{2\left(-L_{4}^{2} d \tau_{0}^{2}+L_{4} c \tau_{0}^{2}-a \tau_{0}^{2}+C\right) \zeta_{0}}{\tau_{1}\left(9 d L_{4}+b\right)} Y+\frac{1}{3 \tau_{1}^{2} L_{5}}\left(-9 a d L_{1} \tau_{0}^{3}\right. \\
& -a b \tau_{0}^{3}+27 d C L_{1} \tau_{0}+3 b C \tau_{0}-27 d L_{1} C_{1}-3 b C_{1}+6 c d L_{4}^{2} \tau_{0}^{3}-3 c^{2} L_{4} \tau_{0}^{3}+3 a c \tau_{0}^{3} \\
& \left.-3 c C \tau_{0}-3 d^{2} L_{4}^{3} \tau_{0}^{3}-3 a d L_{4} \tau_{0}^{3}+3 d C L_{4} \tau_{0}\right)=\left(Y-\alpha_{2}\right)^{2}\left(Y-\alpha_{3}\right),
\end{aligned}
$$

where $\alpha_{2}, \alpha_{3}$ are arbitrary constants. Collecting and letting all coefficients of $Y$ of Equation (27) to be zero, we can get the following results 


$$
\begin{gathered}
\tau_{1}=\frac{1}{L_{1}}, \tau_{0}=\frac{2 \alpha_{2}+\alpha_{3}}{L_{4}}, \\
C=\frac{1}{2 L_{4}^{2} L_{1}}\left(17 L_{1} L_{4}^{2} d \alpha_{2}^{2}+26 L_{1} L_{4}^{2} d \alpha_{2} \alpha_{3}+2 d L_{1} L_{4}^{2} \alpha_{3}^{2}-8 L_{1} L_{4} c \alpha_{1}^{2}-8 L_{1} L_{4} c \alpha_{2} \alpha_{3}\right. \\
\left.-2 L_{1} L_{4} c \alpha_{4}^{2}+L_{4}^{2} b \alpha_{1}^{2}+2 L_{4}^{2} b \alpha_{2} \alpha_{3}+8 L_{1} a \alpha_{2}^{2}+8 L_{1} a \alpha_{2} \alpha_{3}+2 L_{1} a \alpha_{3}^{2}\right), \\
C_{1} \quad \text { (See Appendixes A3) }
\end{gathered}
$$$$
\text { (See Appendixes A3) }
$$

In this family, Equation (18), (19) and (28) lead to get as follows:

$$
\xi_{3}=\zeta_{0}, \xi_{2}=-\left(2 \alpha_{2}+\alpha_{3}\right) \zeta_{0}, \xi_{1}=\alpha_{2}\left(\alpha_{2}+2 \alpha_{3}\right) \zeta_{0}, \xi_{0}=-\alpha_{3} \alpha_{2}^{2} \zeta_{0},
$$

where $\zeta_{0}$ is an arbitrary constant.

When $\alpha_{3}-\alpha_{2}>0$,

$$
\pm\left(\xi-\eta_{0}\right)=\int \frac{\mathrm{d} Y}{\left(Y-\alpha_{2}\right)\left(Y-\alpha_{3}\right)^{\frac{1}{2}}}=-\frac{2}{\sqrt{\alpha_{3}-\alpha_{2}}} \tan ^{-1}\left[\frac{\sqrt{Y-\alpha_{3}}}{\sqrt{\alpha_{3}-\alpha_{2}}}\right],
$$

we can solve for $Y$

$$
Y=\alpha_{3}+\left(\alpha_{3}-\alpha_{2}\right) \tan ^{2}\left[\frac{\sqrt{\alpha_{3}-\alpha_{2}}}{2}\left(\xi-\eta_{0}\right)\right] .
$$

By Equation (31), (29), we can get the rational exact solution of Equation (14) as following

$$
U(\xi)=\frac{2 \alpha_{2}+\alpha_{3}}{L_{4}}+\frac{1}{L_{1}}\left[\alpha_{3}+\left(\alpha_{3}-\alpha_{2}\right) \tan ^{2}\left[\frac{\sqrt{\alpha_{3}-\alpha_{2}}}{2}\left(\xi-\eta_{0}\right)\right]\right] .
$$

With the relation of Equation (7), Equation (1) have the following form of rational travelling wave solution

$$
\begin{aligned}
u(x, t)= & C_{0}\left(\frac{\left(2 \alpha_{2}+\alpha_{3}\right)\left(15 d\left( \pm \frac{-2 c-b+\sqrt{-40 a d+b^{2}+4 b c+4 c^{2}}}{30 d}\right)+b+c\right)}{3 c\left( \pm \frac{-2 c-b+\sqrt{-40 a d+b^{2}+4 b c+4 c^{2}}}{30 d}\right)+2 a}\right. \\
& +\frac{1}{\left( \pm \frac{-2 c-b+\sqrt{-40 a d+b^{2}+4 b c+4 c^{2}}}{30 d}\right)} \\
& \times\left[\alpha_{3}+\left(\alpha_{3}-\alpha_{2}\right) \tan ^{2}\left[\frac{\sqrt{\alpha_{3}-\alpha_{2}}}{2}\left(X-C T-\eta_{0}\right)\right]\right]
\end{aligned}
$$

where $X=C_{2} x+C_{3}, T$ is an arbitrary function of $t$. (Figure 3 and Figure 4)

When $\alpha_{3}-\alpha_{2}<0$,

$$
Y=\alpha_{2}+\left(\alpha_{2}-\alpha_{3}\right) \operatorname{csch}^{2}\left[\frac{\sqrt{\alpha_{3}-\alpha_{2}}}{2}\left(\xi-\eta_{0}\right)\right] \text {. }
$$

Similarly, we can get the exact solution of Equation (1) as follow 


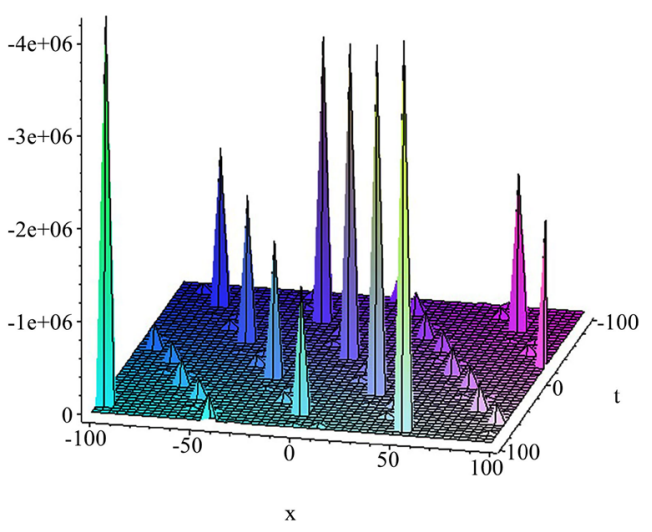

Figure 3. The three-dimensional picture of the exact solution (33) of Equation (1), When $b=3, c=2, \eta_{0}=1, a=d=1$ and $\alpha_{2}=1, \alpha_{3}=5$.

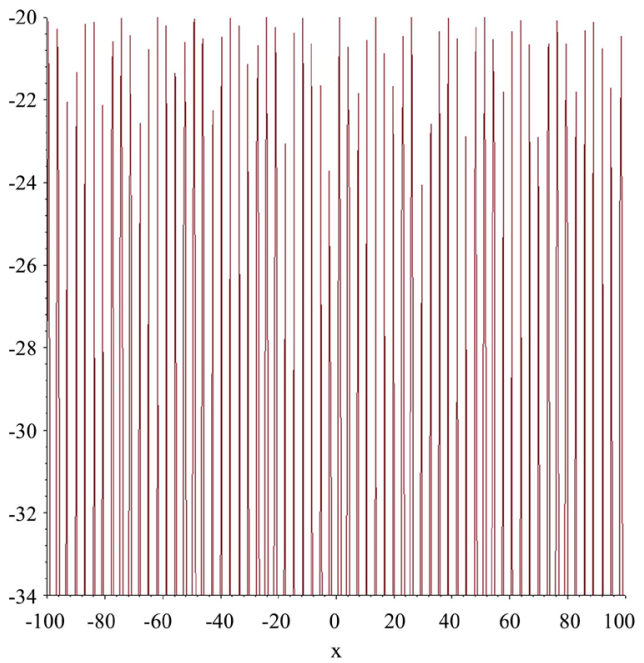

Figure 4. The two-dimensional picture of the equation exact solution (33) of Equation (1), and its projection at $t=0$. When $b=3, c=2, \eta_{0}=1, a=d=1$ and $\alpha_{2}=1, \alpha_{3}=5$.

$$
\begin{aligned}
u(x, t)= & C_{0}\left(\frac{\left(2 \alpha_{2}+\alpha_{3}\right)\left(15 d\left( \pm \frac{-2 c-b+\sqrt{-40 a d+b^{2}+4 b c+4 c^{2}}}{30 d}\right)+b+c\right)}{3 c\left( \pm \frac{-2 c-b+\sqrt{-40 a d+b^{2}+4 b c+4 c^{2}}}{30 d}\right)+2 a}\right. \\
& +\frac{1}{\left( \pm \frac{-2 c-b+\sqrt{-40 a d+b^{2}+4 b c+4 c^{2}}}{30 d}\right)} \\
& \times\left[\alpha_{2}+\left(\alpha_{2}-\alpha_{3}\right) \operatorname{csch}^{2}\left[\frac{\sqrt{\alpha_{2}-\alpha_{3}}}{2}\left(X-C T-\eta_{0}\right)\right]\right]
\end{aligned}
$$

where $X=C_{2} x+C_{3}, T$ is an arbitrary function of $t$ (Figure 5 and Figure 6). 


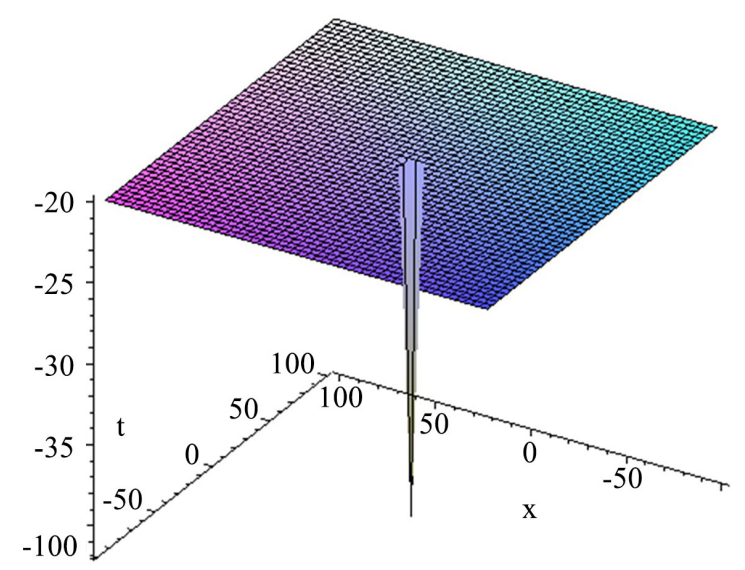

Figure 5. The three-dimensional picture of the exact solution (35) of Equation (1), when $b=3, c=2, a=d=1, \eta_{0}=1$ and $\alpha_{2}=3, \alpha_{3}=1$.

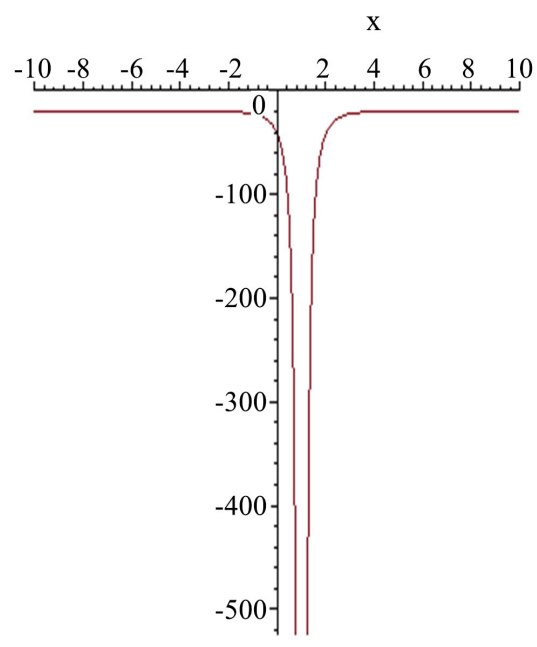

Figure 6. The two-dimensional picture of the equation exact solution (35) of Equation (1), and its projection at $t=0$, when $b=3, c=2, a=d=1, \eta_{0}=1$ and $\alpha_{2}=3, \alpha_{3}=1$.

\section{More Discussion}

In this article, we obtained a series of exact travelling wave solutions of fifth-order KDV equation by the extended trail equation method; according to picking different parameters we can get more exact analytic solutions of nonlinear partial differential equations like fifth-order KDV equation.

The extend trial equation method (ETEM) is proving to play an important role in solving partial differential equations, by using a variety of trail equations, we can construct lots of new types of travelling wave solutions. In this paper, we only considered the following parameters

$$
\varepsilon=0, \theta=3, \delta=1
$$

In a later study we can also study different situations such as $\delta \neq 1$ or $\varepsilon \neq 0$. In this paper, the highest degree of Equations (15) and (16) should be the same, according to balance the highest degree terms of these two Equations (15) and 
(16). So we can take any other parameters that satisfies the following equation

$$
\delta=\theta-\varepsilon-2 .
$$

For example

1) When $\varepsilon=0, \theta=4$ and $\delta=2$.

$$
\left(Y^{\prime}\right)^{2}=\frac{\Phi(Y)}{\Psi(Y)}=\frac{\xi_{0}+\xi_{1} Y+\xi_{2} Y^{2}+\xi_{3} Y^{3}+\xi_{4} Y^{4}}{\zeta_{0}},
$$

and

$$
\begin{gathered}
U=\tau_{0}+\tau_{1} Y+\tau_{2} Y^{2} \\
U^{\prime}=\frac{\left(\tau_{1}+2 \tau_{2} Y\right) \sqrt{\xi_{0}+\xi_{1} Y+\xi_{2} Y^{2}+\xi_{3} Y^{3}+\xi_{4} Y^{4}}}{\sqrt{\zeta_{0}}}
\end{gathered}
$$

$U^{\prime \prime}=$

$$
\begin{gathered}
\frac{12 Y^{4} \tau_{2} \xi_{4}+4 Y^{3} \tau_{1} \xi_{4}+10 Y^{3} \tau_{2} \xi_{3}+3 Y^{2} \tau_{1} \xi_{3}+8 Y^{2} \tau_{2} \xi_{3}+2 Y \tau_{1} \xi_{2}+6 Y \tau_{2} \xi_{1}+\tau_{1} \xi_{1}+4 \tau_{2} \xi_{0}}{2 \zeta_{0}} \\
U^{\prime \prime \prime}=\frac{1}{\sqrt{\zeta_{0}^{3}}} \sqrt{\xi_{0}+\xi_{1} Y+\xi_{2} Y^{2}+\xi_{3} Y^{3}+\xi_{4} Y^{4}}\left(24 Y^{3} \tau_{2} \xi_{4}+6 Y^{2} \tau_{1} \xi_{4}\right. \\
\left.+15 Y^{2} \tau_{2} \xi_{3}+3 Y \tau_{1} \xi_{3}+8 Y \tau_{2} \xi_{3}+\tau_{1} \xi_{2}+3 \tau_{2} \xi_{1}\right)
\end{gathered}
$$

where $\zeta_{0} \xi_{4} \neq 0$.

2) When $\varepsilon=1, \theta=4$ and $\delta=1$.

$$
\left(Y^{\prime}\right)^{2}=\frac{\Phi(Y)}{\Psi(Y)}=\frac{\xi_{0}+\xi_{1} Y+\xi_{2} Y^{2}+\xi_{3} Y^{3}+\xi_{4} Y^{4}}{\zeta_{0}+\zeta_{1} Y},
$$

then

$$
\begin{gathered}
U=\tau_{0}+\tau_{1} Y, \\
U^{\prime}=\tau_{1} \frac{\sqrt{\xi_{0}+\xi_{1} Y+\xi_{2} Y^{2}+\xi_{3} Y^{3}+\xi_{4} Y^{4}}}{\sqrt{\zeta_{0}+\zeta_{1} Y}}, \\
U^{\prime \prime}=\tau_{1}\left(\frac{\xi_{1}+2 \xi_{2} Y+3 \xi_{3} Y^{2}+4 \xi_{4} Y^{3}}{2\left(\zeta_{0}+\zeta_{1} Y\right)^{2}}-\frac{\zeta_{1}\left(\xi_{0}+\xi_{1} Y+\xi_{2} Y^{2}+\xi_{3} Y^{3}+\xi_{4} Y^{4}\right)}{2\left(\zeta_{0}+\zeta_{1} Y\right)^{3}}\right), \\
U^{\prime \prime \prime}=\tau_{1}\left(\frac{1}{2}\left(\zeta_{0}+\zeta_{1} Y\right)^{-\frac{5}{2}}\left(12 \xi_{4} Y^{2}+6 \xi_{3} Y+2 \xi_{2}\right) \sqrt{\xi_{0}+\xi_{1} Y+\xi_{2} Y^{2}+\xi_{3} Y^{3}+\xi_{4} Y^{4}}\right. \\
-\frac{3}{2} \zeta_{1}\left(\zeta_{0}+\zeta_{1} Y\right)^{-\frac{7}{2}}\left(\xi_{1}+2 \xi_{2} Y+3 \xi_{3} Y^{2}+4 \xi_{4} Y^{3}\right) \sqrt{\xi_{0}+\xi_{1} Y+\xi_{2} Y^{2}+\xi_{3} Y^{3}+\xi_{4} Y^{4}} \\
\left.+\frac{3}{2} \zeta_{1}^{2}\left(\zeta_{0}+\zeta_{1} Y\right)^{-\frac{9}{2}}\left(\xi_{0}+\xi_{1} Y+\xi_{2} Y^{2}+\xi_{3} Y^{3}+\xi_{4} Y^{4}\right)^{\frac{3}{2}}\right),
\end{gathered}
$$

where $\xi_{\theta}$ and $\zeta_{\varepsilon}$ are arbitrary positive integers to be determined in later calculations.

In later studies, when studying other nonlinear partial differential equations, we can also obtain the relationship of parameters through balancing the highest degree terms of these equations, select parameters and apply the Extend trial equation method (ETEM) to solve the exact solution of the equation. 


\section{Conclusion}

In this letter, the ETEM has been successfully applied to construct exact traveling wave solutions for fifth-order KDV equation. Then, the solutions of corresponding nonlinear partial differential equations with variable coefficients are obtained by the equivalence transformation given in Section 2. In later studies, many solutions of variable coefficient PDEs can be considered in the same procedure. Generally, for tackling exact solutions to vc-PDEs are difficult, the results in this paper provide a useful supplement to the existing literature. Moreover, the equivalence transformation and improved ETEM can be used to other types of vc-PDEs in mathematical physics.

\section{Conflicts of Interest}

The authors declare no conflicts of interest regarding the publication of this paper.

\section{References}

[1] Qureshi, M.I., Quraishi, K.A. and Srivastava, H.M. (2008) Some Hypergeometric Summation Formulas and Series Identities Associated with Exponential and Trigonometric Functions. Integral Transforms \& Special Functions, 19, 267-276. https://doi.org/10.1080/10652460801896024

[2] Yu, H. (2016) On Generalized Trigonometric Functions and Series of Rational Functions. Journal of Number Theory, 180, 512-532. https://doi.org/10.1016/j.jnt.2017.05.015

[3] Zhang, Z., Zhong, J., Dou, S., Liu, J., Peng, D. and Gao, T. (2013) A New Method to Construct Travelling Wave Solutions for the Klein-Gord-Zakharov Equations. Romanian Journal of Physics, 58, 766-777.

[4] Miao, X. and Zhang, X. (2011) The Modified (G'G)-Expansion Method and Traveling Wave Solutions of Nonlinear the Perturbed Nonlinear Schrödinger's Equation with Kerr Law Nonlinearity. Communications in Nonlinear Science and Numerical Simulation, 16, 4259-4267. https://doi.org/10.1016/j.cnsns.2011.03.032

[5] Feng, Z. and Wang, X. (2003) The First Integral Method to the Two-Dimensional Burgers-Korteweg-de Vries Equation. Physics Letters A, 308, 173-178. https://doi.org/10.1016/S0375-9601(03)00016-1

[6] Eslami, M. and Rezazadeh, H. (2015) The First Integral Method for Wu-Zhang System with Conformable Time-Fractional Derivative. Calcolo, 53, 475-485. https://doi.org/10.1007/s10092-015-0158-8

[7] Qian, S. and Tian, L. (2007) Modification of the Clarkson-Kruskal Direct Method for a Coupled System. Chinese Physics Letters, 24, 2720-2723. https://doi.org/10.1088/0256-307X/24/10/002

[8] Chen, M. and Liu, X.Q. (2011) Exact Solutions and Conservation Laws of the Konopelchenko-Dubrovsky Equations. Pure \& Applied Mathematics, 27, 533-532.

[9] Yuan, Q. and Lei, Y. (2011) Symmetry Groups and New Exact Solutions of (2+1)Dimensional Broer-Kaup-Kupershmidt (BKK) System. International Conference on Multimedia Technology.

[10] He, J. and Abdou, M. (2007) New Periodic Solutions for Nonlinear Evolution Equations Using Exp-Function Method. Chaos, Solitons \& Fractals, 34, 1421-1429. 
https://doi.org/10.1016/j.chaos.2006.05.072

[11] Kudryashov, N.A. and Loguinova, N.B. (2009) Be Careful with the Exp-Function Method. Communications in Nonlinear Science \& Numerical Simulation, 14, 1881 1890. https://doi.org/10.1016/j.cnsns.2008.07.021

[12] Hossain, A.K.M.K.S., Akbar, M.A. and Wazwaz, A.M. (2017) Closed Form Solutions of Complex Wave Equations via the Modified Simple Equation Method. Cogent Physics, 4, Article ID: 1312751. https://doi.org/10.1080/23311940.2017.1312751

[13] Akter, J. and Ali Akbar, M. (2015) Exact Solutions to the Benney-Luke Equation and the Phi-4 Equations by Using Modified Simple Equation Method. Results in Physics, 5, 125-130. https://doi.org/10.1016/j.rinp.2015.01.008

[14] Jiao, X.Y. and Lou, S.Y. (2009) Approximate Direct Reduction Method: Infinite Series Reductions to the Perturbed mKdV Equation. Chinese Physics B, 18, 3611 3615. https://doi.org/10.1088/1674-1056/18/9/001

[15] Taghizadeh, N., Mirzazadeh, M. and Farahrooz, F. (2011) Exact Travelling Wave Solutions of the Coupled Klein-Gordon Equation by the Infinite Series Method. Applications and Applied Mathematics, 6, 1964-1972.

[16] Liu, H.Z., Li, J.B. and Liu, L. (2010) Lie Symmetry Analysis, Optimal Systems and Exact Solutions to the Fifth-Order KdV Types of Equations. Journal of Mathematical Analysis and Applications, 368, 551-558. https://doi.org/10.1016/j.jmaa.2010.03.026

[17] Wang, G.W., Liu, X.Q. and Zhang, Y.Y. (2013) Symmetry Reduction, Exact Solutions and Conservation Laws of a New Fifth-Order Nonlinear Integrable Equation. Communications in Nonlinear Science and Numerical Simulation, 18, 2313-2320. https://doi.org/10.1016/j.cnsns.2012.12.003

[18] Liu, H.Z. (2015) Painlevé Test, Generalized Symmetries, Bäcklund Transformations and Exact Solutions to the Third-Order Burgers' Equations. Journal of Statistical Physics, 158, 433-446. https://doi.org/10.1007/s10955-014-1130-8

[19] Liu, H.Z. and Li, J.B. (2012) Painlevé Analysis, Complete Lie Group Classifications and Exact Solutions to the Time-Dependent Coefficients Gardner Types of Equations. Nonlinear Dynamics, 80, 515-527. https://doi.org/10.1007/s11071-014-1885-0

[20] Sahoo, S. and Ray, S.S. (2017) Analysis of Lie Symmetries with Conservation Laws for the $(3+1)$ Dimensional Time-Fractional mKdV-ZK Equation in Ion-Acoustic Waves. Nonlinear Dynamics, 90, 1105-1113. https://doi.org/10.1007/s11071-017-3712-x

[21] Xin, X.P. (2018) Non-Local Symmetries and Exact Solutions of Nonlinear Development Equations. Journal of Liaocheng University (Natural Science), 32, 15-20. (In Chinese)

[22] Ekici, M., Mirzazadeh, M., Sonmezoglu, A., et al. (2017) Nematicons in Liquid Crystals by Extended Trial Equation Method. Journal of Nonlinear Optical Physics \& Materials, 26, Article ID: 1750005. https://doi.org/10.1142/S0218863517500059

[23] Ekici, M., Mirzazadeh, M., Sonmezoglu, A., et al. (2017) Optical Solitons with Anti-Cubic Nonlinearity by Extended Trial Equation Method. Optik-International Journal for Light and Electron Optics, 136, 368-373. https://doi.org/10.1016/j.ijleo.2017.02.004

[24] Abbasbandy, S. and Zakaria, F.S. (2008) Soliton Solutions for the Fifth-Order KdV Equation with the Homotopy Analysis Method. Nonlinear Dynamics, 51, 83-87. https://doi.org/10.1007/s11071-006-9193-y 
[25] Bridges, T.J., Derks, G. and Gottwald, G. (2002) Stability and Instability of Solitary Waves of the Fifth-Order KdV Equation: A Numerical Framework. Physica DNonlinear Phenomena, 172, 190-216. https://doi.org/10.1016/S0167-2789(02)00655-3

[26] Leite Freire, I. and Santos Sampaio, J.C. (2012) Corrigendum: Nonlinear Self-Adjointness of a Generalized Fifth-Order KdV Equation. Journal of Physics A Mathematical \& Theoretical, 45, Article ID: 119502.

https://doi.org/10.1088/1751-8113/45/11/119502

[27] Triki, H. and Biswas, A. (2011) Soliton Solutions for a Generalized Fifth-Order KdV Equation with T-Dependent Coefficients. Waves in Random \& Complex Media, 21, 151-160. https://doi.org/10.1080/17455030.2010.539632 


\section{Appendixes}

$$
\begin{aligned}
& \xi_{0}=2 \zeta_{0}\left(-6 d \tau_{0}^{3} L_{2}^{2} a^{2}-L_{2}^{3} a b \tau_{0}^{3}+3 C L_{2}^{3} b \tau_{0}-27 C_{1} L_{1} L_{2}^{3} d-9 c^{3} \tau_{0}^{3} L_{2}^{2} L_{1}\right. \\
& +3 a c \tau_{0}^{3} L_{2}^{3}-6 a c^{2} \tau_{0}^{3} L_{2}^{2}-3 c \tau_{0} L_{2}^{3} C-81 d^{2} \tau_{0}^{3} L_{1}^{3} c^{3}-24 d^{2} \tau_{0}^{3} a^{3} \\
& -3 C_{1} L_{2}^{3} b+6 d \tau_{0} C L_{2}^{2} a-9 L_{1} L_{2}^{3} d a \tau_{0}^{3}+27 C L_{1} L_{2}^{3} d \tau_{0}+54 c^{3} \tau_{0}^{3} L_{2} L_{1}^{2} d \\
& +24 c \tau_{0}^{3} L_{2} d a^{2}-162 d^{2} \tau_{0}^{3} L_{1}^{2} c^{2} a-108 d^{2} \tau_{0}^{3} L_{1} c a^{2}+72 c^{2} \tau_{0}^{3} L_{1} L_{2} a d \\
& \left.-9 d \tau_{0}^{3} L_{1} L_{2}^{2} a c+9 d \tau_{0} C L_{1} L_{2}^{2} c\right) /\left(3 L_{2}^{3} \tau_{1}^{2} L_{3}\right) . \\
& C_{1}=\alpha_{1}^{3}\left(10935 L_{1}^{5} L_{2} d^{2} c^{2}+2187 L_{1}^{5} d^{2} c^{3}-1458 L_{2}^{4} L_{2}^{2} d c^{2}+14580 L_{1}^{4} L_{2} d^{2} a c\right. \\
& +1944 L_{1}^{4} L_{2} d b c^{2}-729 L_{1}^{4} L_{2} d c^{3}+4374 L_{1}^{4} d^{2} a c^{2}+243 L_{1}^{4} d b c^{3} \\
& +324 L_{1}^{3} L_{2}^{3} d a-972 L_{1}^{3} L_{2}^{2} d a c-162 L_{1}^{3} L_{2}^{2} b c^{2}+4860 L_{1}^{3} L_{2} d^{2} a^{2} \\
& +2592 L_{1}^{3} L_{2} a b c d-972 L_{1}^{3} L_{2} a d c^{2}+81 L_{1}^{3} L_{2} b^{2} c^{2}-81 L_{1}^{3} L_{2} b c^{3} \\
& +2916 L_{1}^{3} a^{2} d^{2} c+486 L_{1}^{3} a d b c^{2}+27 L_{1}^{3} L_{3} c^{3}+36 L_{1}^{2} L_{2}^{3} a b-108 L_{1}^{2} L_{2}^{2} a b c \\
& +864 L_{1}^{2} L_{2} a^{2} b d-324 L_{1}^{2} L_{2} a^{2} d c+108 L_{1}^{2} L_{2} a b^{2} c-108 L_{1}^{2} L_{2} a b c^{2} \\
& +648 L_{1}^{2} a^{3} d^{2}+324 L_{1}^{2} a^{2} b c d+54 L_{1}^{2} L_{3} a c^{2}+36 L_{1} L_{2} a^{2} b^{2}-36 L_{1} L_{2} a^{2} b c \\
& \left.+72 L_{1} d a^{3} b+36 L_{1} L_{3} a^{2} c+8 L_{3} a^{3}\right) /\left(2 L_{1}^{2}\left(3 L_{1} c+2 a\right)^{3}\left(9 d L_{1}+b\right)\right) . \\
& C_{1}=\frac{1}{6 L_{1}^{2} L_{4}^{3}\left(9 d L_{1}+b\right)}\left(156 L_{1}^{2} d L_{4}^{2} b \alpha_{2}^{3}+432 L_{1}^{3} a d \alpha_{2}^{2} \alpha_{3}+135 L_{4}^{3} d^{2} L_{1}^{2} \alpha_{2}^{2} \alpha_{3}\right. \\
& +48 L_{1}^{2} a b \alpha_{2}^{2} \alpha_{3}+1863 L_{1}^{3} d^{2} L_{4}^{2} \alpha_{2}^{2} \alpha_{3}+810 \alpha_{2} L_{1}^{3} d^{2} L_{4}^{2} \alpha_{3}^{2}+216 \alpha_{2} L_{1}^{3} a d \alpha_{3}^{2} \\
& +24 \alpha_{2} L_{1}^{2} a b \alpha_{3}^{2}+54 L_{4}^{3} d^{2} L_{1}^{2} \alpha_{3}^{2} \alpha_{2}+6 L_{1}^{2} L_{4}^{2} b d \alpha_{3}^{3}+6 L_{1} L_{4}^{3} d b \alpha_{2}^{3} \\
& +15 L_{1} L_{4}^{2} b^{2} \alpha_{2}^{2} \alpha_{3}+6 L_{1} L_{4}^{2} b^{2} \alpha_{3}^{2} \alpha_{2}-432 L_{1}^{3} d c L_{4} \alpha_{2}^{3}-54 L_{4}^{2} d c L_{1}^{2} \alpha_{2}^{3} \\
& -48 L_{1}^{2} L_{4} b c \alpha_{2}^{3}-54 L_{1}^{3} d c L_{4} \alpha_{3}^{3}-6 L_{1}^{2} L_{4} b c \alpha_{3}^{3}-6 L_{1} L_{4}^{2} b c \alpha_{2}^{3}+3 \alpha_{2}^{2} \alpha_{3} L_{4}^{3} L_{3} \\
& +6 L_{1} L_{4}^{2} b^{2} \alpha_{2}^{3}+918 L_{1}^{3} d^{2} L_{4}^{2} \alpha_{2}^{3}+288 L_{1}^{3} a d \alpha_{2}^{3}+54 L_{4}^{3} d^{2} L_{1}^{2} \alpha_{2}^{3}+32 L_{1}^{2} a b \alpha_{2}^{3} \\
& +54 L_{1}^{3} d^{2} L_{4}^{2} \alpha_{3}^{3}+36 L_{1}^{3} a d \alpha_{3}^{3}+4 L_{1}^{2} a b \alpha_{3}^{3}+144 \alpha_{2} L_{1}^{2} L_{4}^{2} b d \alpha_{3}^{2} \\
& +342 L_{1}^{2} b d L_{1}^{2} L_{4}^{2} \alpha_{2}^{2} \alpha_{3}-36 \alpha_{2} L_{1}^{2} L_{4} b c \alpha_{3}^{2}-648 L_{1}^{3} d c L_{4} \alpha_{2}^{2} \alpha_{3} \\
& -135 L_{4}^{2} d c L_{1}^{2} \alpha_{2}^{2} \alpha_{3}-72 L_{1}^{2} L_{4} b c \alpha_{2}^{2} \alpha_{3}-54 L_{4}^{2} d c L_{1}^{2} \alpha_{3}^{2} \alpha_{2}-15 L_{1} L_{4}^{2} b c \alpha_{2}^{2} \alpha_{3} \\
& \left.-6 L_{1} L_{4}^{2} b c \alpha_{3}^{2} \alpha_{2}-324 \alpha_{3}^{2} \alpha_{2} L_{1}^{3} L_{4} d+15 L_{1} L_{4}^{3} b d \alpha_{2}^{2} \alpha_{3}+6 L_{1} L_{4}^{3} b d \alpha_{3}^{2} \alpha_{2}\right) \text {. }
\end{aligned}
$$

